\title{
Mesenchymal stem cell therapy induces glucocorticoid synthesis in colonic mucosa and suppresses radiation-activated T cells: new insights into MSC immunomodulation
}

\author{
R Bessout, A Sémont, C Demarquay, A Charcosset, M Benderitter and N Mathieu
}

Non-neoplastic tissues around an abdomino-pelvic tumor can be damaged by the radiotherapy protocol, leading to chronic gastrointestinal complications that affect the quality of life with substantial mortality. Stem cell-based approaches using immunosuppressive bone marrow mesenchymal stem cells (MSCs) are promising cell therapy tools. In a rat model of radiation proctitis, we evidenced that a single MSC injection reduces colonic mucosa damages induced by ionizing radiation with improvement of the re-epithelization process for up to 21 days. Immune cell infiltrate and inflammatory molecule expressions in the colonic mucosa were investigated. We report that MSC therapy specifically reduces T-cell infiltration and proliferation, and increases apoptosis of radiation-activated $\mathrm{T}$ cells. We assessed the underlying molecular mechanisms and found that interleukin-10 and regulatory $\mathrm{T}$ lymphocytes are not involved in the immunosuppressive process in this model. However, an increased level of corticosterone secretion and HSD11b1 (11ß-hydroxysteroid dehydrogenase type 1)-steroidogenic enzyme expression was detected in colonic mucosa 21 days after MSC treatment. Moreover, blocking the glucocorticoid (GC) receptor using the RU486 molecule statistically enhances the allogenic lymphocyte proliferation inhibited by MSCs in vitro and abrogates the mucosal protection induced by MSC treatment in vivo. Using the irradiation model, we found evidence for a new MSC immunosuppressive mechanism involving GCs.

\section{INTRODUCTION}

Radiation therapy is an established part of the treatment of both primary and recurrent pelvic and abdominal malignancies, including colorectal, urologic, and gynecologic cancers. Although the benefit of radiotherapy on tumor regression is well-established, the damage to healthy non-neoplastic tissues may be severe and still remains the dose-limiting factor in cancer treatment. It is estimated that $90 \%$ of patients who undergo pelvic radiotherapy develop acute side effects with permanent changes in bowel habits. Within 10 years, $5-10 \%$ of the patients develop severe chronic side effects such as rectal bleeding, occlusion, tenesmus, and diarrhea, associated with substantial morbidity and mortality (for review, see ref. 1). The growing number of cases declared each year and the specific complex symptoms have led some specialists to talk of a new disease termed "Pelvic-radiation disease". ${ }^{2}$ Understanding the mechanisms involved and the discovery of new therapies have increasingly become a priority.

The early response following ionizing radiation, mainly studied in the intestine, has been characterized as acute inflammation with a high level of cytokines and chemokines secretion within the first hours. Radiation-induced apoptosis later leads to transient mucosal atrophy with loss in bowel barrier function. The resultant epithelial dysfunction leads to nutrient and fluid loss, whereas the associated increase in intestinal permeability to gut pathogens exacerbates the mucosal inflammation with dense infiltration of the lamina propria by inflammatory cells, mainly neutrophils and macrophages. ${ }^{3}$ The endothelial compartment is also affected by the radiation exposure, leading to increased vascular permeability contributing to immune cell infiltration into injured tissue. Delayed effects of intestinal radiation toxicity are

Institut de Radioprotection et de Sûreté Nucléaire (IRSN), PRP-HOM, SRBE, LRTE, Fontenay-aux-Roses, France. Correspondence: N Mathieu (noelle.mathieu@irsn.fr) Received 2 April 2013; accepted 16 September 2013; published online 30 October 2013. doi:10.1038/mi.2013.85 
characterized by an abnormally sustained repair process that can lead to transmural fibrosis. The mechanism of radiation injury is a continuous process starting immediately after irradiation. There is increasing evidence, suggesting that some of the risk of late toxicity is related to the degree of acute toxicity, termed "consequential late effects", and recent clinical data support the hypothesis that cumulative acute symptoms predict late problems more effectively than peak acute changes. ${ }^{4}$ The treatments usually applied to manage acute side effects are only symptomatic, so searching for treatments limiting the acute radiation toxicity in order to reduce late side effects makes sense.

In order to achieve a combinatorial approach to disease treatment, some investigators have considered the use of stem cells as therapeutic tools. Stem cell-based approaches using mesenchymal stem cells (MSCs) are promising, as numerous studies suggest their immunosuppressive and regenerative capacities. MSC-mediated inhibition of the immune response is a complex mechanism that could involve low immunogenicity, the ability to modify maturation and the function of antigen-presenting cells, as well as an alteration in the cytokine secretion of naive dendritic cells, natural killer cells and effector $\mathrm{T}$ cells. ${ }^{5-8}$ These findings have encouraged the use of MSCs in acute graft-versus-host disease ${ }^{9}$ and autoimmune diseases ${ }^{10,11}$ to induce tolerance.

Following pelvic radiotherapy, the colorectum is more commonly injured because of its fixed position in the pelvis. In Sprague-Dawley rats, we generated radiation-induced lesions similar to those seen in patients suffering from side effects after pelvic radiotherapy. ${ }^{12}$ We characterized immune cell infiltration and modification after MSC treatment. We assessed the molecular mechanisms of MSC immunosuppression by analyzing anti-inflammatory molecule secretion in irradiated mucosa and their effects on downstream T-cell signaling.

\section{RESULTS}

\section{MSC treatment reduces epithelial damages induced by colorectal irradiation}

The epithelial damages induced by ionizing radiation were analyzed on colonic circular histological sections realized every $1,500 \mu \mathrm{m}$ throughout $3 \mathrm{~cm}$ of the distal colon (Figure 1). After 7 days, radio-induced apoptosis leads to epithelial cell depletion within the crypts (Figure 1b), on the majority of the $3-\mathrm{cm}$ irradiated window. Thereafter (14 days), a worsening of the lesion occurs with loss of crypt architecture (Figure 1c). Twenty-one and 28 days after the radiation exposure (Figure 1d,e), the mucosa presents profound ulceration zones and zones with few regenerative crypts. The benefit of MSC treatment was quantified using an epithelial injury score, taking into account epithelial atypia and mucosal ulceration. We have previously shown that MSC injection improves colonic mucosa after 7 and 14 days. ${ }^{13}$ In this study, we analyzed whether the benefit of MSCs can endure beyond. After 21 days, in irradiated-MSC-treated animals we observed a significant decrease of the epithelial injury score value (Figure 1f) with a decrease of the ulcerated zones (Figure 1g) and an increase of regenerating crypts (Figure 1h) compared with irradiated animals (Figure 1d). Indeed, 21 days after irradiation we observed at the margin of the ulcerated areas, zones with improvement of the re-epithelization process compared with those irradiated without treatment. Quantification of the proliferating epithelial cells was assessed on histological slides stained with PCNA (proliferating cell nuclear antigen) antibody. The number of proliferating cells per crypt is statistically lower in irradiated animals compared with controls $(26.9 \pm 0.5$ vs. $30.9 \pm 0.4, P<0.001)$. After MSC infusion into irradiated rats, the number of proliferating cells per crypt reaches $35.2 \pm 0.9(P<0.001$ vs. control and $P<0.001$ vs. Irr). Twenty-eight days after irradiation, histopathologic analysis failed to provide evidence of any MSC therapeutic benefit on the epithelial injury. These results demonstrate that the MSC treatment induces benefits for up to 21 days. Moreover, they suggest that a single MSC injection is not sufficient to sustainably improve severe radiation-induced mucosal damages.

\section{MSC treatment specifically decreases activated T lymphocytes induced by colorectal irradiation}

To quantify immune cell infiltration over time following localized colorectal irradiation and its modulation after MSC treatment, we used flow cytometry analysis on leukocytes isolated from the colon. We observed that irradiation leads to a six-fold increase in the percentage of neutrophils in leukocyte infiltrate, 7 days after irradiation, compared with controls. This increase persists for 21 and 28 days after irradiation. No modification in the neutrophil infiltrate observed after irradiation can be detected after MSC injection at any of the different times analyzed (D7, D21, and D28; Figure 2a). We also analyzed neutrophil infiltration, 21 days after irradiation, by immunohistochemistry using antibodies directed against myeloperoxidase. In the same way, no modification of radiation-induced neutrophil infiltration can be evidenced by scoring after MSC therapy (Figure 2b, left panel). Two other cells of innate immunity, macrophages and mastocytes, were analyzed on histological slides. The results given in Figure $\mathbf{2 b}$ show a statistic increase in macrophages and mastocytes in mucosa 21 days after irradiation (middle and right panels). However, no modification was observed after MSC treatment. Previous reports demonstrated that irradiation induces proinflammatory cytokine secretion, in particular interleukin (IL)$1 \beta$, IL6, and tumor necrosis factor- $\alpha$, in insulted areas as early as the first hours. ${ }^{14,15}$ Subsequent secretion of these molecules after irradiation, which could be involved in late tissue damage, has never been studied. We therefore analyzed, 21 days after irradiation, the secretion of cytokines and chemoattractants produced mainly by innate immune cells and their modulation after MSC treatment. IL6 and tumor necrosis factor- $\alpha$ cannot be detected by enzyme-linked immuno-assay on whole colonic mucosa protein extracts after localized irradiation. IL1 $\beta$, macrophages inflammatory protein-2, and IL8 are secreted in significant quantities after irradiation and neither of them decreases after MSC therapy (Figure 2c). Altogether, these data 

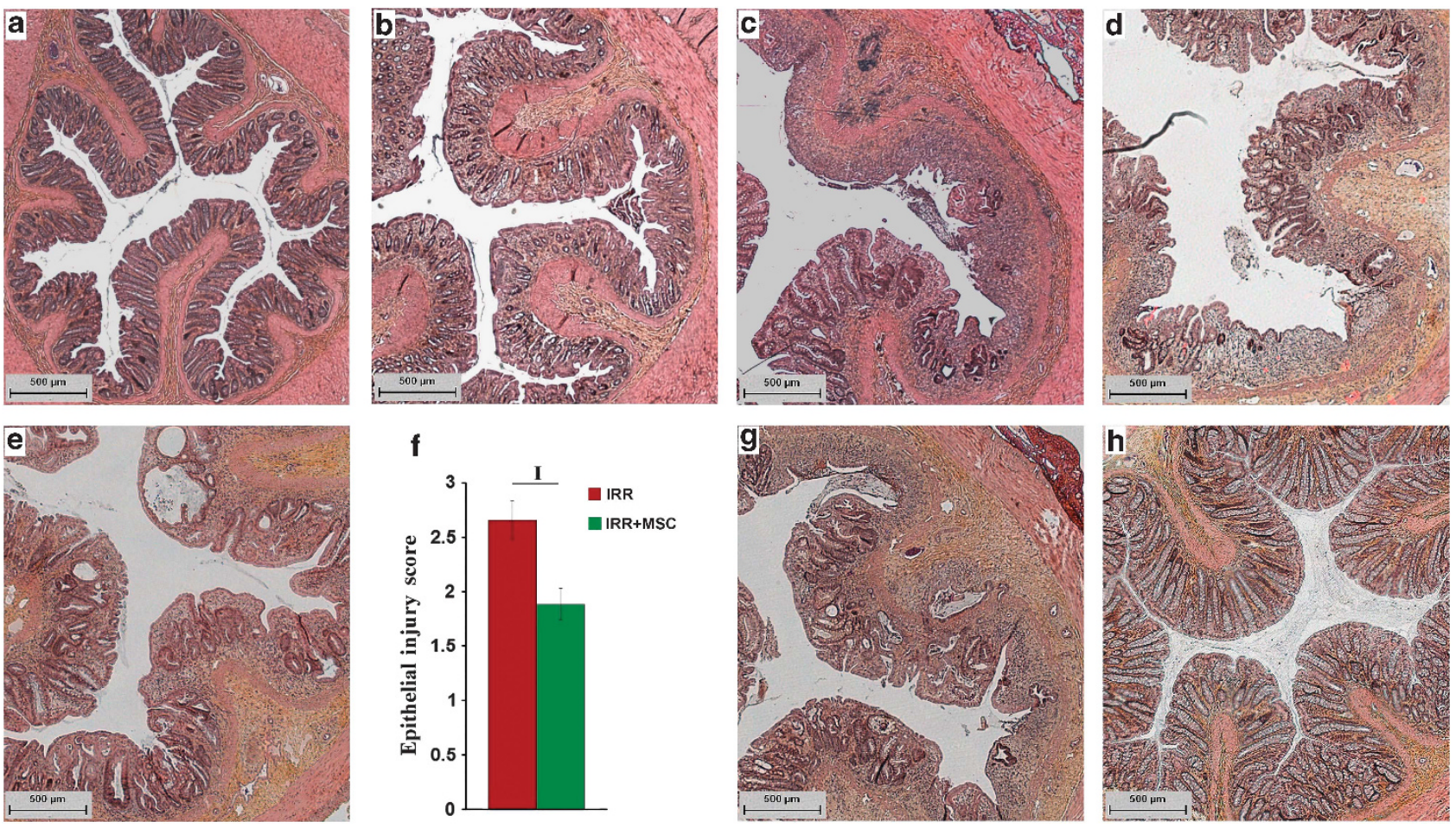

Figure 1 Mesenchymal stem cell (MSC) treatment reduces epithelial damages induced by colorectal irradiation until 21 days after irradiation. Representative histological pictures of colonic mucosa from (a) control and irradiated rats after (b) 7 days, (c) 14 days, (d) 21 days, and (e) 28 days. (f) Epithelial injury score 21 days after irradiation in irradiated (red histogram) and irradiated-MSC-treated rats (green histogram). On a scale from 1 to 3 , epithelial atypia and mucosal ulceration were evaluated on hematoxylin-eosin-saffron-colored histological sections. The scoring of these variables was as follows: 0 , none; 1 , epithelial radiation injury affecting $<25 \%$ of the circular colon section; 2 , epithelial radiation injury affecting between 25 and $50 \%$ of the circular colon section; and 3 , epithelial radiation injury affecting $>50 \%$ of the circular colon section. Representative histological pictures of mucosa from irradiated-MSC-treated rats after 21 days in ulcerated zones $(\mathbf{g})$ and in regenerative zones (h). Results are expressed as mean \pm s.e.m. $(n=6$ at least for each group). I, $P<0.05$ versus irradiated animals.

demonstrate that MSC treatment does not modify either the number or the cytokine secretion abilities of innate cells.

In irradiated mucosa, B, T, and natural killer cells were also quantified. The results show a statistical increase only for $\mathrm{T}$ cells as early as 7 days. This increase, observed after irradiation, endures for up to 28 days for $\mathrm{CD} 4$ helper and 21 days for $\mathrm{CD} 8$ cytotoxic $\mathrm{T}$ cells. After MSC infusion, the percentage of T cells is statistically lower at 21 and 28 days compared with the irradiated group (Figure 2d). Twenty-eight days after irradiation, we observed that even if the number of CD8 in irradiated animals was no different from the untreated group, a statistical decrease was detected after MSC treatment (Figure 2d). The immunosuppressive effect of MSCs on T cells was also observed in the peripheral area (draining mesenteric lymph nodes (MLNs) and blood; data not shown) after localized irradiation of the colon.

\section{Cellular mechanisms of the MSC immunosuppressive effect after colorectal irradiation}

T-lymphocyte activation was assessed 21 days after irradiation using the IL2 receptor (CD25) marker on total T lymphocytes from the colon, MLNs, and blood. Flow cytometry results show that the percentage of $\mathrm{CD} 25$ activation markers increases by $116.7 \%$ in the colon, $16.78 \%$ in MLNs, and $41.7 \%$ in blood in leukocytes isolated from rats after irradiation. Either in the colon or in the peripheral area, MSC infusion after irradiation decreases the percentage of $\mathrm{CD} 25$ activation markers to the levels found in untreated animals (Figure 3a). To further investigate the activation status in CD4 and CD8 subsets, we analyzed $\mathrm{CD} 4 / \mathrm{CD} 25$ and $\mathrm{CD} 8 / \mathrm{CD} 25$ double-positive cells in MLNs of rats from different groups (Figure $3 \mathbf{b}$ ). The results demonstrate that MSC therapy decreases the CD25 activation markers induced by irradiation in MLNs to the same extent for CD4 and for CD8 T-cell subsets. This result was confirmed by quantitative real-time $\mathrm{PCR}$ on isolated $\mathrm{CD} 4$ and $\mathrm{CD} 8$ lymphocytes using the CD69 marker (data not shown). We also assessed T-cell proliferation using a bromodeoxyuridinebased proliferation assay on sorted CD4 and CD8 populations. Corroborating CD25 marker expression, irradiation leads to a statistical increase of CD4 and CD8 T-cell proliferation, and MSC treatment reduces their ability to proliferate (Figure 3c). Regulation of T-cell apoptosis is an important mechanism controlling the number of activated $\mathrm{T}$ cells. We therefore examined apoptosis by Annexin $\mathrm{V}$ staining on ex vivo EdU (5ethynyl-2'-deoxyuridine)-incorporated CD4- and CD8-sorted $\mathrm{T}$ cells from untreated, irradiated, and irradiated-MSC-treated animals. The results show a statistical increase in apoptosis (1.5 times more) of proliferating CD8 T cells from irradiatedMSC-treated rats compared with irradiated rats (Figure 3d). However, no statistical difference is detected among proliferating $\mathrm{CD} 4 \mathrm{~T}$ cells from the various groups. 


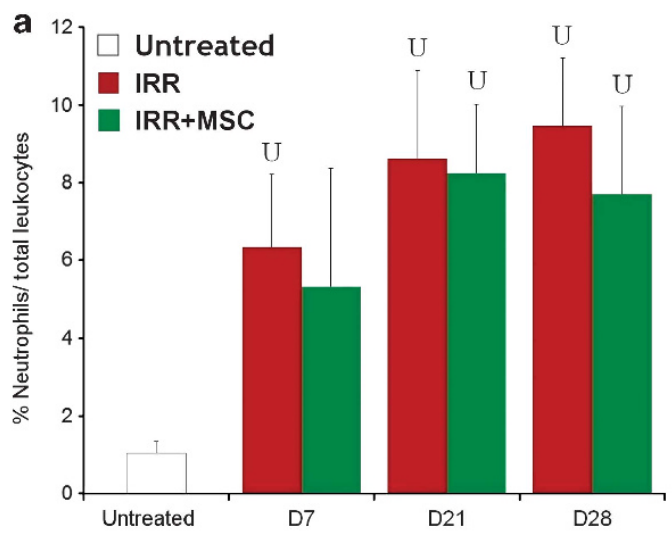

b


Figure 2 Mesenchymal stem cell (MSC) treatment induces no modification of innate immune cell recruitment nor of cytokine secretion profile but decreases T-lymphocytes infiltrate. (a) Quantification of the neutrophils by flow cytometry on total leukocytes isolated from the colon. (b) Immunohistochemistry analyses were realized 21 days after irradiation using myeloperoxidase (MPO) antibody and score of neutrophils infiltration (left panel). Middle and right panels: macrophages (CD68) and mastocytes (toluidine blue) staining. (c) Twenty-one days after irradiation, cytokine secretion was measured by enzyme-linked immuno-assay (ELISA). (d) Time-course analysis of the percentage of CD4 (CD3 + CD4 +) and CD8 (CD3 + CD8 +) T cells in total colonic leucocytes. All results are expressed as mean \pm s.e.m. $U, P<0.05$ versus untreated animals. $I, P<0.05$ versus irradiated animals. 
a
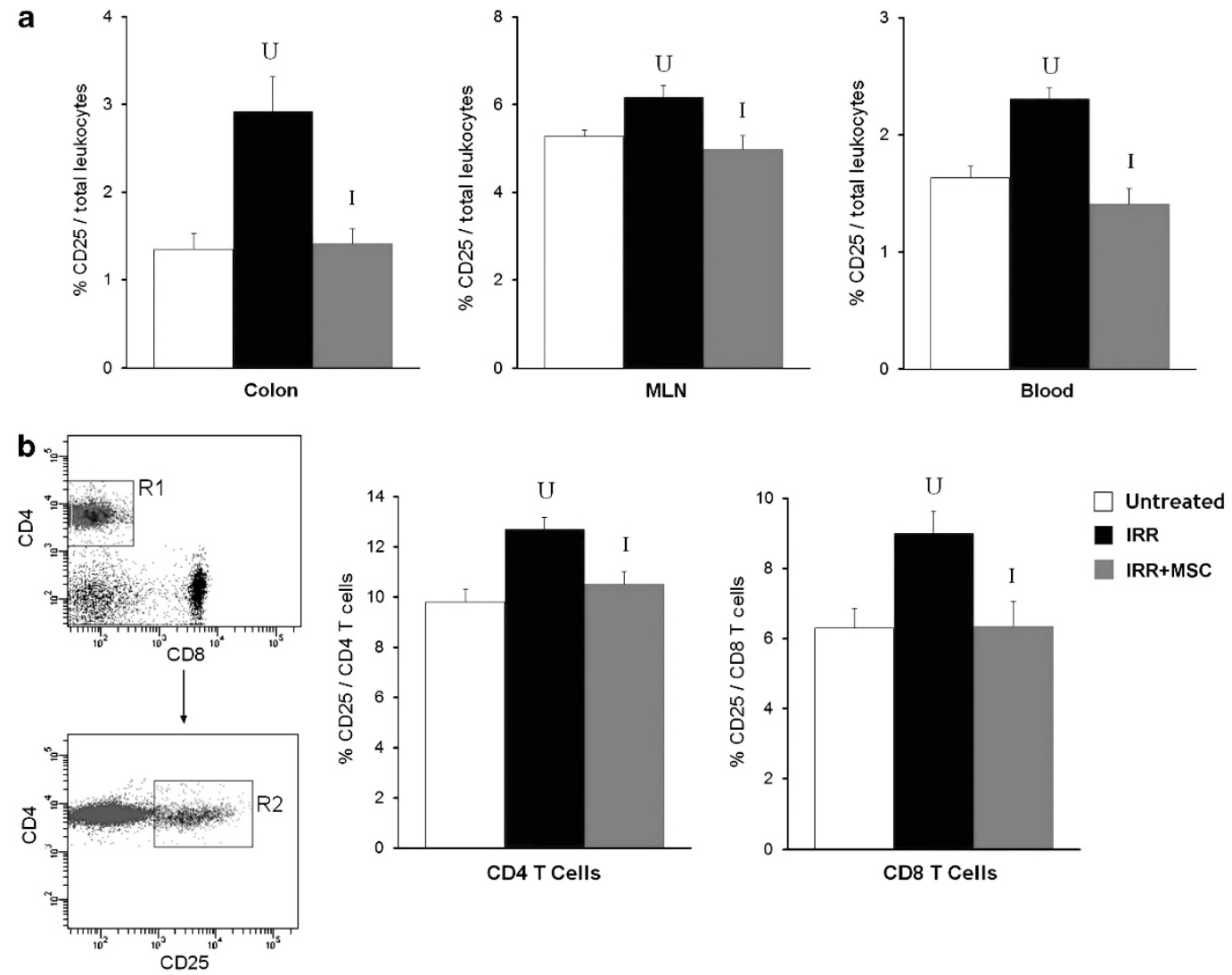

CD8 T Cells
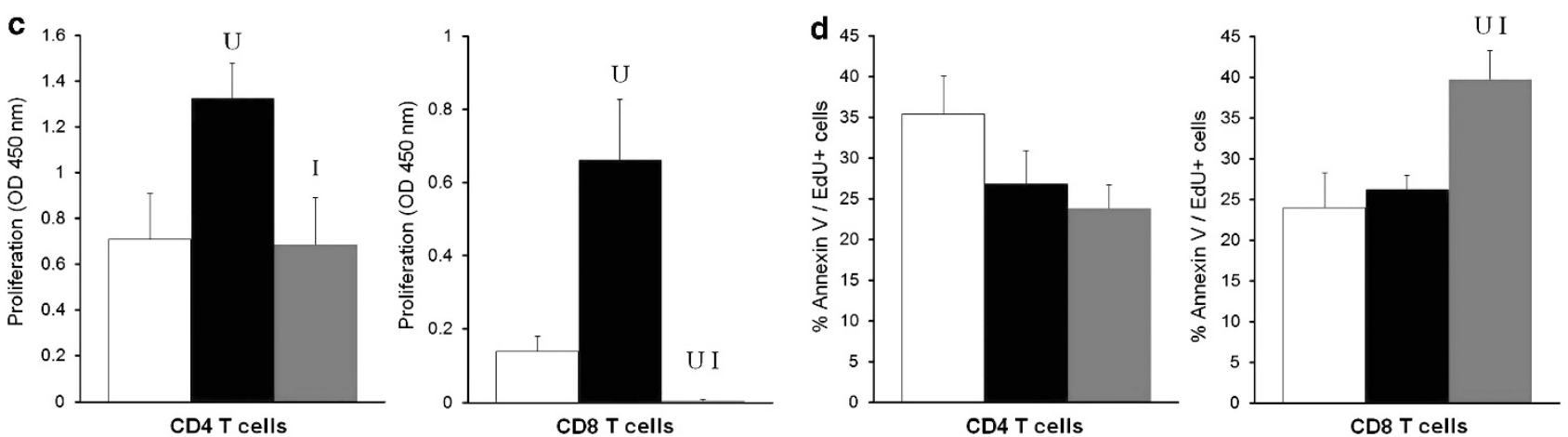

Figure 3 Effect of mesenchymal stem cell (MSC) treatment on radiation-activated T lymphocytes 21 days after irradiation. (a) Flow cytometry quantification of CD25-positive cells in colon, mesenteric lymph nodes (MLNs) and blood 21 days after irradiation. (b) Percentage of CD4 + CD25 + and CD8 + CD25 + positive cells among total MLN cells. (c) CD4- and CD8-sorted lymphocytes from MLNs were induced to proliferate and assessed for bromodeoxyuridine (BrdU) proliferation assay. (d) Measurement of apoptosis on proliferating cells was assessed ex vivo on purified CD4 and CD8 T cells. All results are expressed as mean \pm s.e.m. $U, P<0.05$ versus untreated animals. I, $P<0.05$ versus irradiated animals.

\section{MSC infusion increases local corticosterone secretion after colorectal irradiation}

An increasing number of in vivo studies have demonstrated that IL10-secreting regulatory T lymphocytes (T-Reg) are involved in the therapeutic effect of MSCs in inflammatory bowel diseases. ${ }^{5,16,17}$ However, depending on the disease model, the molecular mechanisms of immunosuppression induced by MSCs seem to vary. ${ }^{8,18}$ To study the immunosuppressive mechanisms of MSCs in an irradiation context, IL10 antiinflammatory cytokine secretion was analyzed after MSC therapy. The results shown in Figure 4a do not demonstrate any increase of IL10 in colonic mucosa. Corroborating this result, we showed that T-Reg cells detected by the CD4/CD25/ FoxP3 triple staining do not increase after MSC treatment in irradiated rats compared with controls (Figure 4b). IL10secreting T-Reg are unlikely to be involved in the T-cell modulation induced by MSC treatment after irradiation.

Therefore, we investigated a new mechanism of MSC immunosuppression in the irradiation model. The intestine contains a large number of immune cells to protect the huge surface of epithelial cells. The immune system is in constant contact with food antigens and commensal bacteria, as well as potentially dangerous bacteria, viruses, and parasites. The intestinal mucosa contains high levels of immunosuppressive 

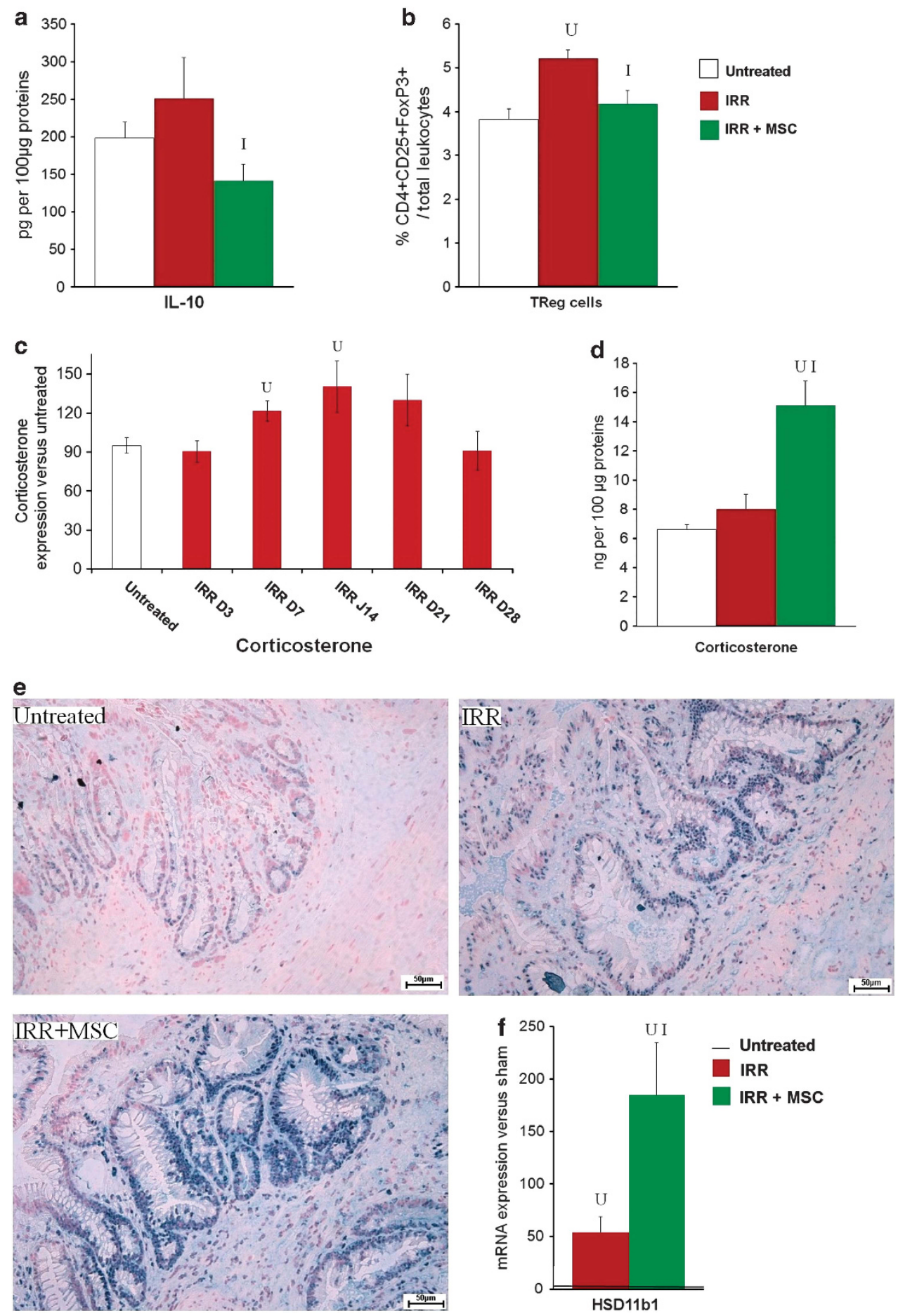

Figure 4 Mesenchymal stem cell (MSC) treatment increases corticosterone secretion in irradiated colon. (a) Enzyme-linked immuno-assay (ELISA) measurement of interleukin (IL10) secretion in colonic mucosa. (b) Fluorescence-activated cell sorting (FACS) detection of CD4 + CD25 + FoxP3 + triple-positive cells into total mesenteric lymph node (MLN) cells. Experiments were done 21 days after radiation exposure. (c) Time-dependent ELISA measurement of corticosterone secretion in colonic mucosa after $3,7,14,21$, and 28 days after irradiation compared with untreated animals. (d) ELISA measurement of corticosterone in colonic mucosa of untreated rats (white bar), irradiated rats (red bar), and irradiated-MSC-treated rats (green bar) after 21 days. (e) Representative pictures of $11 \beta$-hydroxysteroid dehydrogenase type 1 (HSD11b1) immunohistochemistry (blue staining) on colonic sections from untreated, irradiated, and irradiated-MSC-treated animals. (f) Relative HSD11b1 mRNA expression in mucosa at 21 days of irradiated and irradiated-MSC-treated rats. Results were normalized with the GAPDH housekeeping gene and standardized to the level of untreated animals (untreated animals expression $=1$ ). Experiments were done 21 days after radiation exposure and results are expressed as mean \pm s.e.m. Animal number for each group is at least six. $U, P<0.05$ versus untreated animals. $I, P<0.05$ versus irradiated animals. 
cytokines, but additional mechanisms exist to maintain a confined immune response, such as glucocorticoids (GCs). GCs are indeed secreted by mucosal cells and studies have demonstrated their important role in intestinal T-cell regulation. ${ }^{19}$ We therefore investigated whether the immunosuppressive action of MSC therapy could be due to an increase in GCs. We analyzed the corticosterone molecule, which is the major GCs in rats, in the adrenal glands, blood, and colon. In adrenal glands and blood, no modification was detected in irradiated and irradiated-MSC-treated rats (data not shown). However, in colonic mucosa we demonstrated that the corticosterone rate increases statistically 7 and 14 days after irradiation compared with untreated animals. Next, we observed a decrease at day 21 , with the rate returning to the control level at day 28 (Figure 4c). Seven and 14 days after irradiation, MSC infusion generates no further increase in GC secretion. However, 21 days after irradiation a twofold increase of the corticosterone level is observed in irradiated animals after MSC infusion compared with irradiated rats (Figure 4d). This effect does not last beyond 28 days. We also analyzed the expression of HSD11b1 (11 $\beta$-hydroxysteroid dehydrogenase type 1) in colonic tissue 21 days after irradiation (Figure 4e,f). This enzyme, involved in the metabolism of corticosterone, is important in local GC-level regulation and is expressed in the colon. Immunohistochemistry analyses on normal colonic mucosa showed HSD11b1 expression in the lamina propria and epithelial cells (Figure 4e, top left panel) as previously described. $^{19}$ After irradiation and irradiation with MSC treatment, we observed a dramatic increase in HSD11b1 expression (Figure 4e, top right and bottom panels). Quantification of HSD11b1 expression using real-time PCR Figure $4 \mathrm{f}$ demonstrated a statistical increase in its expression after irradiation by 53 -fold, and an even higher increase after MSC treatment (183-fold).

\section{Signal transduction induced by GCs on T cells from irradiated rats: in vivo and ex vivo analyses}

MSC treatment after irradiation decreases T-cell proliferation by reducing their activation and increases $\mathrm{CD} 8 \mathrm{~T}$-cell apoptosis. The effects of GCs on these functions are widely recognized and can be achieved through the classic genomic pathway (gene regulation at transcriptional level) or the non-genomic pathway. In our irradiation model, we cannot detect proinflammatory cytokine secretions such as IL2 and interferon $-\gamma$ in whole-protein extracts from irradiated mucosa. Moreover, transcriptional upregulation of IL10, which is usually the target of GCs leading to an increase in T-Reg, is not observed after MSC therapy (Figure 4a,b). Hence, GCinduced cytokine regulation by the genomic pathway is unlikely to be the main mechanism involved in our model. Nongenomic GC activities are obviously not uniform, but Löwenberg et al..$^{20}$ demonstrated in $\mathrm{T}$ cells that the GC receptor (GR) is an integral and essential part of the T-cell receptor (TCR) complex. They demonstrated that GC treatment, by inducing GR nuclear translocation, breaks up the TCR complex, leading to impaired TCR signaling
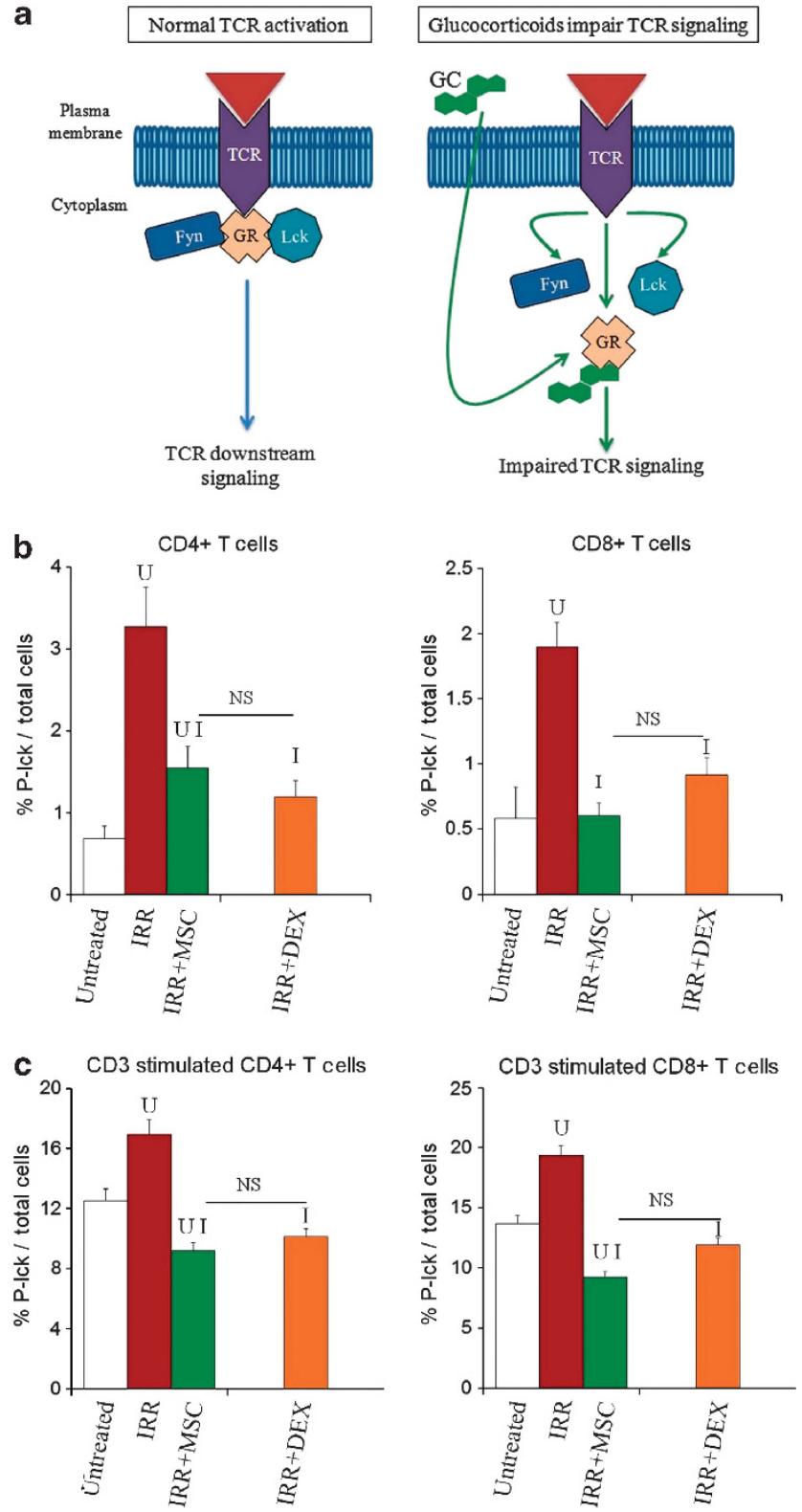

Figure 5 Mesenchymal stem cell (MSC) or dexamethasone treatment impairs T-cell receptor (TCR) signaling onto CD4 and CD8 T cells from irradiated animals. (a) Schematic representation of glucocorticoid receptor (GR) interactions with TCR adapted from Löwenberg et al. ${ }^{20}(\mathbf{b}, \mathbf{c})$ Percentage of phosphorylated p56lck (P-lck)-positive cells among total CD4 and CD8 T cells. Twenty-one days after irradiation exposure, sorted CD4 and CD8 T cells from different groups (white bars = untreated, red bars $=$ irradiated, and green bars $=\mathrm{irr}+\mathrm{MSC}$ treatment) were incubated with culture medium (b) or culture medium with CD3 antibody (c), fixed onto microscope slides, and stained for phosphorylated p56lck kinase (P-lck). Effect of synthetic corticosterone (DEX) on TCR signaling was verified ex vivo on T lymphocytes from irradiated rats incubated with $1 \mu \mathrm{m}$ dexamethasone. Percentage of P-lck-positive cells is represented by orange bars $(\mathbf{b}, \mathbf{c})$. All experiments were done 21 days after radiation exposure and results are expressed as mean \pm s.e.m., and are representative of two independent experiments with four animals per group for each experiment. $U, P<0.05$ versus untreated animals. I, $P<0.05$ versus irradiated animals. NS, not significant; $P>0.05$ versus MSC-treated animals.

(Figure 5a). We therefore analyzed the impact of irradiation, MSC treatment, and GCs on CD4 and CD8 TCR signaling, by immunofluorescence staining using the phosphorylated p56lck 
antibody. Irradiation induces a dramatic increase in the percentage of $\mathrm{CD} 4$ and $\mathrm{CD} 8$ phosphorylated p56lck-positive cells compared with untreated animals (Figure 5b). After MSC treatment, the percentage of phosphorylated p56lck-positive cells among CD4- and CD8-sorted cells decreases statistically, with a return to the untreated level for CD8 T cells. We also tested ex vivo the ability of dexamethasone, a synthetic GC, to inhibit TCR signaling of CD4 and CD8 T cells purified from irradiated rats. We found that the incubation of $\mathrm{T}$ cells from irradiated rats with dexamethasone reduces the percentage of phosphorylated p56lck-positive lymphocytes compared with lymphocytes without dexamethasone incubation (Figure 5b). This result demonstrates that a synthetic GC can reduce TCR signaling in lymphocytes activated by irradiation. We then assessed the ability of sorted $\mathrm{T}$ cells to respond to subsequent direct TCR stimulation using CD3 agonist antibodies. This experiment also demonstrated a decrease in TCR signaling in the T-cell population purified from irradiated-MSC-treated rats compared with irradiated rats (Figure 5c). Taken together, these data suggest that the increased level of corticosterone in colonic mucosa after MSC treatment can contribute to TCR unresponsiveness and decreased T-cell activation.

\section{Infused MSCs express HSD11b1, produce GCs in culture and the RU486 molecule alleviates MSC-induced immunosuppression}

We have previously demonstrated MSC engraftment in the irradiated intestine, ${ }^{21,22}$ suggesting that MSC benefits are mediated via soluble factors as stated in various studies (for a review, see ref. 23). We therefore investigated the ability of infused MSCs to express enzymes involved in GC metabolism (HSD11b1, CYP11A1, and CYP11B) and to produce GCs in culture. As shown in Figure 6a, corticosterone is detected in MSC culture supernatant and in MSC total protein extracts. PCR analysis showed high expression of HSD11b1, indeed its expression level is similar to the housekeeping GAPDH gene. CYP11A1 and CYP11B1 enzymes are less expressed than HSD11b1 (Figure 6b). Expression of HSD11b1 was confirmed at the protein level by immunofluorescence on cultured MSCs (Figure 6c). Moreover, we analyzed whether these enzymes are modulated by stimuli induced after disruption of the epithelial barrier following irradiation $(\mathrm{H} 2 \mathrm{O} 2$, PAM3CSK4, lipopolysaccharide, and flagellin). We also tested interferon- $\gamma$ stimulation, which has been described as having an important role in MSC immunosuppression. ${ }^{24}$ As shown in Figure 6d, CYP11A1 expression does not increase after stimulation, whereas CYP11B1 increases after interferon- $\gamma$ stimulation. We determined that HSD11b1 expression, which is already highly expressed, increases to an even greater extent after Toll-like receptor (TLR) ligand stimulations.

To analyze the involvement of GCs in MSC immunosuppression, we added a GR antagonist, RU486, into a classical MLR (mixed lymphocytes reaction) inhibition test by MSCs. First, we tested various concentrations of RU486 $\left(10^{-5} \mathrm{M}\right.$, $10^{-6} \mathrm{M}$, and $10^{-7} \mathrm{M}$ ) on allogenic lymphocyte proliferation. As previously published, we found that the highest concentration of RU486 $\left(10^{-5} \mathrm{M}\right)$ inhibits lymphocyte proliferation. On the other hand, the use of the lowest RU486 concentration $\left(10^{-7} \mathrm{M}\right)$ is not able to restore allogenic proliferation induced by $1 \mu \mathrm{m}$ dexamethasone. Therefore, experiments have been realized with RU486 at $10^{-6} \mathrm{M}$. As expected, MSCs exert a dose-dependent inhibitory effect on MLR reaction, decreasing allogenic lymphocyte proliferation between $15 \%$ for the lower dose $\left(1.10^{4}\right.$ MSCs) and $67 \%$ for the higher one $\left(1.10^{5}\right.$ MSCs; Figure 6e). When RU486 is added to the MLR/MSC reaction (allogenic lymphocytes and MSCs), lymphocyte proliferation increases statistically for each MSC rate. The percentage of restoration varies between 14.67 and $29.98 \%$ compared with MLR/MSCs without RU486. Thus, MSC-induced inhibition of allogenic lymphocyte proliferation is alleviated by GR blockade determining the involvement of the GC pathway in the MSC immunosuppressive effect.

\section{Blocking GC action with the RU486 molecule prevents MSC benefits on mucosal lesions}

To determine the direct involvement of MSCs on the GCmediated protection from radiation damages to the mucosa, we inhibited the GC action in vivo using the RU486 molecule. RU486 or vehicle was injected subcutaneously every 3 days into untreated, irradiated, and irradiated-MSC-treated rats during 21 days. Histological analyses (Figure 7a,b) and epithelial scoring (Figure 7c) determine an increase of mucosal lesions in irradiated rats injected with the GR blocking molecule (RU486) compared with irradiated rats injected with the vehicle $\left({ }^{\$} P<0.001\right)$. Colitis cystica profunda structures increase substantially after RU486 injections on irradiated and irradiated-MSC-treated animals (Figure 7a,b, middle and right panels). We determined that the number of sections presenting colitis cystica profunda structures increases by threefold in these groups compared with the vehicleinjected groups (Figure $7 \mathbf{d d},{ }^{*} P<0.001$ ). However, RU486 injections have no deleterious effect on colonic mucosa in control rats (Figure 7a,b, left panels). Animals injected with vehicle demonstrate a statistical MSC-induced benefit on mucosal lesions induced by irradiation (Figure 7a,c). Conversely, in irradiated animals injected with RU486, the MSC treatment is not able to reduce mucosal lesions (Figure $7 \mathbf{b}, \mathbf{c})$. This result demonstrates that the mucosal protection induced by MSCs following irradiation is lost when GC action is blocked.

\section{DISCUSSION}

Ionizing radiation produces a burst of free radicals, which not only causes DNA damage but also alters proteins, lipids, carbohydrates, and complex molecules. In organs, this contributes to direct tissue injury, as well as cytokine synthesis and recruitment of inflammatory cells. Cellular damage induced by ionizing radiation generally ceases within hours following the insult, whereas the inflammatory response accumulates, amplifies tissue injuries, and reduces the efficacy of the regenerative process. Therefore, the inflammatory reaction after irradiation is presumed to have a pivotal role in the development of pelvic-radiation disease, and it is important to 

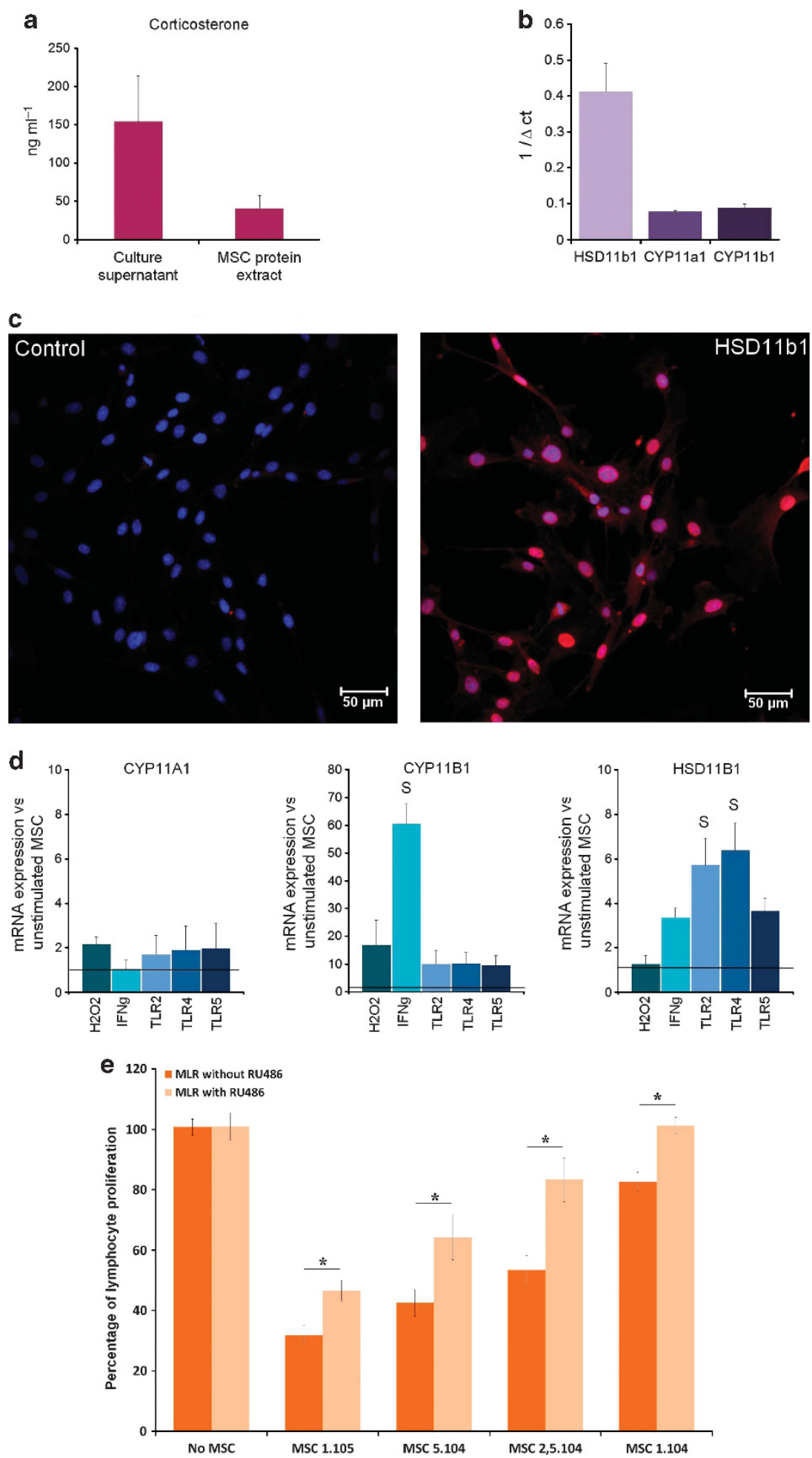

Figure 6 Mesenchymal stem cell (MSC) tolerogenic effects are partly mediated in vitro via secretion of corticosterone. In vitro cultured MSCs produce corticosterone and express HSD11b1. (a) Quantification of rat corticosterone by enzyme-linked immuno-assay (ELISA) on 24h cultured MSC supernatant and MSC protein extract. (b) Messenger RNA (mRNA) expression of enzymes involved in glucocorticoid (GC) metabolism normalized with GAPDH in cultured MSCs. (c) Confocal microscopy imaging of 11 $\beta$-hydroxysteroid dehydrogenase type 1 (HSD11b1) expression on cultured MSCs. (d) Relative mRNA expression of enzymes following MSC stimulation normalized with GAPDH and standardized to unstimulated MSCs (unstimulated MSCs =1). Results are expressed as mean \pm s.e.m. and experiments were done in triplicate on MSCs derived from three different samples. $U, P<0.05$ versus untreated animals. (e) Blocking GC action with the RU486 molecule alleviates MSC-induced immunosuppression in vitro. MSCs significantly inhibit mixed lymphocyte reaction in a dose-dependent manner. This inhibition is partially alleviated in the presence of RU486 (10 $\left.{ }^{-6} \mathrm{M}\right)$. Results represent the mean \pm s.e.m. and are representative of seven independent experiments. 
a

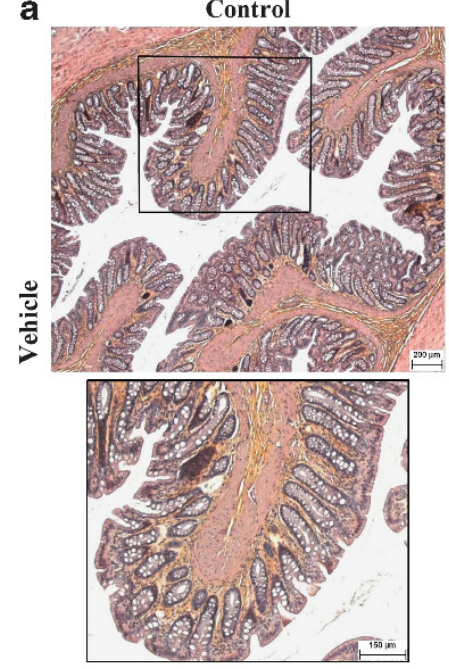

b
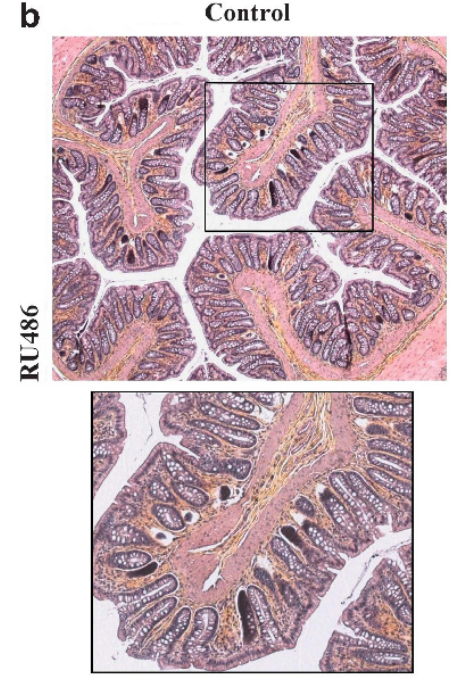

IRR

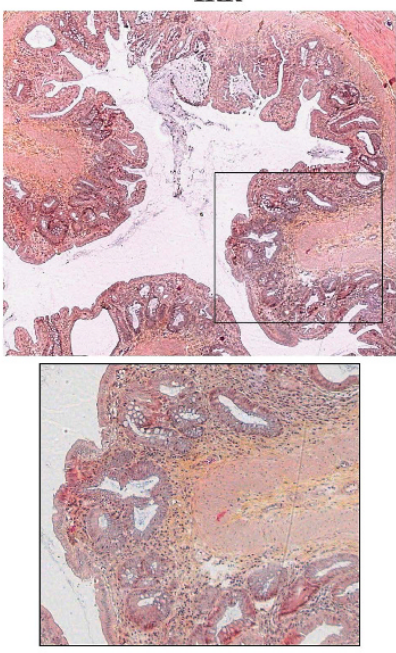

IRR
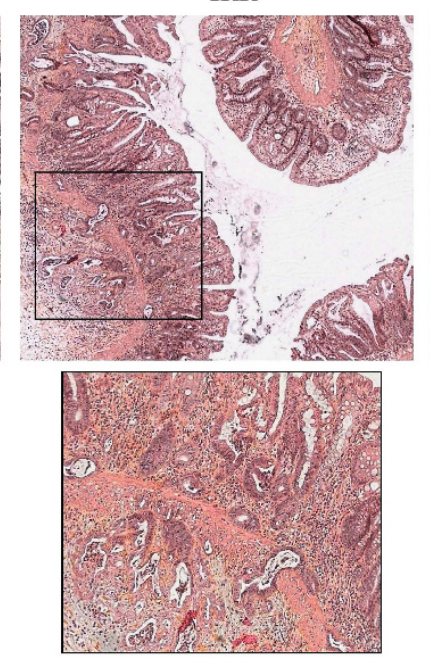

IRR + MSC

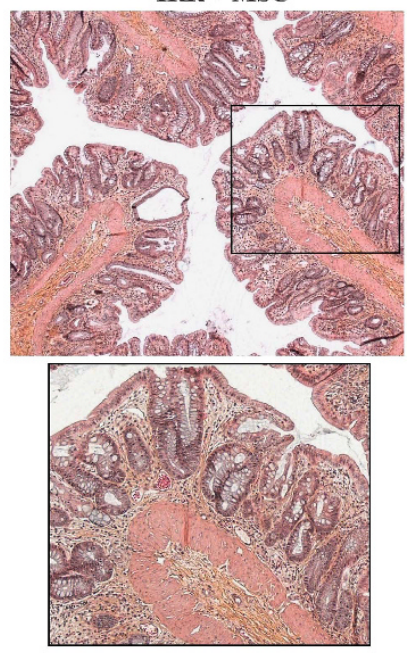

IRR + MSC
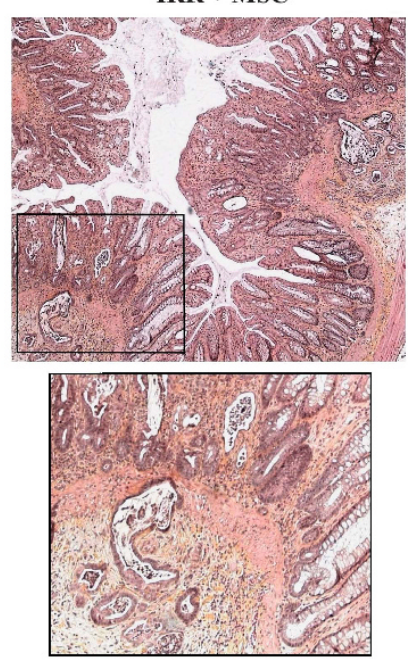
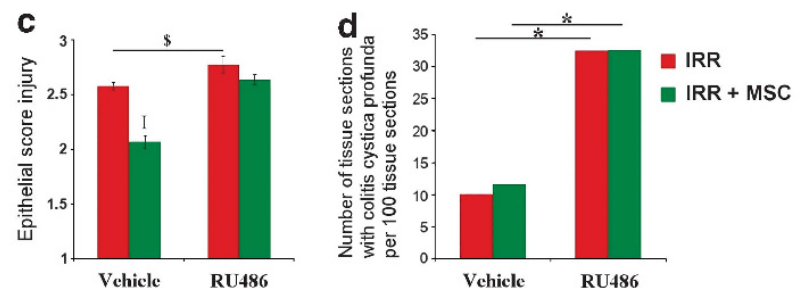

Figure 7 Blocking glucocorticoid (GC) action with the RU486 molecule alleviates mesenchymal stem cell (MSC)-induced protection of mucosal lesions in vivo. $(\mathbf{a}, \mathbf{b})$ Representative histological pictures of colonic mucosa from controls, irradiated, and irradiated-MSC-treated rats injected with vehicle (a) or RU486 (b) during 21 days (two magnifications were presented). (c) Epithelial injury score. Mucosal lesions were increased after RU486 injections in irradiated animals $\left({ }^{\$} P<0.001\right)$ and MSC treatment reduces epithelial lesions in vehicle-injected animals $(I, P<0.05$ versus irradiated animals). RU486 injections alleviate MSC-induced benefit induced by irradiation. (d) Number of tissue sections with colitis cystica profunda for 100 tissue sections analyzed $\left({ }^{*} P<0.01\right)$. All analyses were realized after 21 days. Results are expressed as mean \pm s.e.m. ( $n=8$ for each group).

determine the immune cells involved in order to mitigate the inflammatory process. Our study characterized the composition of innate immune cell infiltrate in colonic mucosa for up to 4 weeks in our experimental colorectal irradiation rat model and the results are in agreement with the human cell composition observed after late irradiation damage. Innate immune cell infiltrate has been largely described after irradiation. In human colonic tissues, histopathology analysis exhibits inflammatory cell infiltration in the lamina propria, with a prevalence of neutrophils, eosinophilic granulocytes, and macrophages. ${ }^{2}$ Mast cell hyperplasia has also been described in colonic mucosa associated with late irradiation damage. ${ }^{14}$ In intestinal inflammatory pathologies, it is largely admitted that chronic activated $\mathrm{T}$ cells contribute to mucosal damage. Little data is available regarding late irradiation damage. In animal models, a T-cell imbalance has been demonstrated. ${ }^{25}$ However, 
depending on the studied irradiation models, there is either no modification or a decrease of CD4 T cells in the intestine. ${ }^{26,27}$ The present findings, using quantitative flow cytometry analysis, demonstrate the persistent infiltrate of CD4 and CD8 T cells in colonic mucosa up to 4 weeks after colorectal irradiation. We also describe a statistical increase in the T-cell percentage in the peripheral area after local irradiation, demonstrating their ability to continuously recirculate. $\mathrm{T}$ lymphocytes from irradiated rats are more activated as measured by the increase of CD25 and CD69 markers, and by their ability to proliferate in culture compared with control lymphocytes. Radiation treatment of tumors modifies and increases the peptide repertoire (tumor peptides but also self-antigen peptides) presented by major histocompatibility complex class I to cytotoxic CD8 T lymphocytes. $^{28}$ It has been demonstrated that cytotoxic CD8 T lymphocytes are activated by this unrecognized repertoire composed of specific irradiated peptides, which are very useful in anti-tumor immunotherapy. ${ }^{29}$ However, self-antigens may also be presented when irradiation affects healthy tissues, and cytotoxic CD8 T lymphocytes may therefore attack and destroy colonic cells, perpetuating tissue lesions. Besides upregulation of $\mathrm{CMH}$ class I, irradiation also induces secretory, adhesion, and co-stimulatory molecules, as well as Fas expression on hematological or non-hematological cells. ${ }^{30}$ Together, these data may explain the activation process of CD8 cytotoxic and CD4 helper cells, as well as of antigen-presenting cells after irradiation.

MSCs are mesoderm-derived cells with the ability to extensively proliferate and differentiate in various cell lineages. In addition to these primordial characteristics for tissue repair, MSCs have also been shown to hold a potent anti-inflammatory effect on immune disorders such as graft-versus-host disease ${ }^{31}$ or Crohn's disease. ${ }^{32}$ In vitro studies have demonstrated the impact of MSCs on practically all immune cell subtypes (for a review, see ref. 33). In this study, we analyzed in vivo the ability of MSCs to suppress inflammation induced by ionizing radiation, and we determined the cellular and molecular mechanisms involved. We also analyzed the benefit of MSC therapy on mucosal damages. The results demonstrate statistical improvement of the mucosal structure. However, analysis of the innate immune cells demonstrates that their number and secretion abilities are not modified by MSC therapy. We were unable to exclude a modification of the maturation status or function of innate cells associated with the T-cell suppression mechanism by MSCs in our irradiation model. It has been demonstrated that MSCs are able to reprogram macrophages to become immunosuppressive $^{34}$ and in vivo depletion of CD11b + macrophages abolishes the MSC immunosuppressive effect on $\mathrm{T}$ cells. ${ }^{17}$ Further investigations will be necessary to address this aspect of inflammation induced by irradiation. The number and proliferation ability of both CD4 and CD8 T cells return to control level and the apoptosis of proliferating CD8 T cells increases following MSC treatment. The MSC effect is observed as early as 7 days but becomes statistically relevant at 21 days. Altogether, these results demonstrate the immunosuppressive effect of MSC therapy on $\mathrm{T}$ cells activated by irradiation and are in accordance with studies obtained in other in vivo models. ${ }^{6,8}$
The precise molecular mechanisms of the MSC effect on $\mathrm{T}$-cell modulation remain unresolved. Various in vitro studies have demonstrated that the immunosuppressive effect is mediated through several inducible soluble factors such as transforming growth factor- $\beta$, hepatocyte growth factor, IL10, prostaglandin E2, and indoleamine 2,3-dioxygenase. ${ }^{35}$ Using specific antagonists, partial reversion of the MSC inhibitory effect on T-cell proliferation was observed, suggesting that various molecules are involved in the immunosuppressive abilities of MSCs. Our study demonstrates secretion of corticosterone, a powerful anti-inflammatory molecule, by cultured MSCs associated with the expression of enzymes involved in the corticosterone metabolism. We also observed, using a GR antagonist in MLR/MSC reaction, the restoration of $\sim 30 \%$ of the proliferative capacities of allogeneic lymphocytes. These results highlight the involvement of GCs in the immunosuppressive abilities of MSCs.

An increasing number of in vivo studies have demonstrated that the IL10 anti-inflammatory molecule associated with an increased number of T-Reg is involved in the therapeutic effect of MSCs. ${ }^{5,16,17,34}$ However, according to the model the MSC immunosuppressive effect may be T-Reg independent. ${ }^{6,7,18}$ Although we can easily detect IL10 in MSC culture supernatant, we cannot detect an increase of IL10 secretion or T-Reg cells in irradiated colonic mucosa after MSC infusion. After colorectal irradiation, we found that the MSC treatment increases corticosterone secretion and HSD11b1 expression in colonic mucosa, which is associated with T-cell modulation. These results reveal a new molecular mechanism of the MSC immunosuppressive action in vivo. Such an MSC effect has never been described. The effects of GCs on T-cell immunosuppression are widely recognized and can be achieved through the classic genomic pathway or the non-genomic pathway. ${ }^{20}$ Investigations of the molecular action mechanism of MSC immunosuppression by signal transduction analysis suggest the involvement of the non-genomic pathways in T-cell immunosuppression in the irradiation context. The GC genomic pathway is known to induce IL10 secretion and inhibit proinflammatory cytokines such as IL2 and interferon- $\gamma$; it can therefore be hypothesized that GC synthesis is also involved in the MSC immunosuppressive mechanism in other models. This study highlights the molecular mechanism of the MSC immunosuppressive effect involving GC, valuable in radiationinduced inflammation but possibly also involved in other inflammatory bowel diseases. We also demonstrated in vitro that stimuli induced by irradiation $\left(\mathrm{H}_{2} \mathrm{O}_{2}\right.$ or TLR ligands) induce an increase in enzymes involved in corticosterone secretion by MSCs. This notion that MSCs must be stimulated to exert their immunosuppressive capacities has already been described $^{24}$ and is of interest for the purpose of enhancing the therapeutic effect of MSCs.

Various studies have demonstrated MSC engraftment in tissue following damages (the brain, heart, lung, and kidney). It has been hypothesized that MSC engraft in tissue through the same mechanism as leukocytes. Indeed, inflammatory molecules were expressed by the tissue following damages. 
Furthermore, many of the molecules involved in the tethering, rolling, adhesion, and transmigration of leukocytes from the bloodstream into the tissue are known to be expressed on MSCs. Ionizing radiation triggers inflammation but also stem/progenitor cell apoptosis, highly limiting the regenerative ability of mucosa, and leading to chronic damages. We have previously described MSC engraftment in the intestine of immunodeficient mice ${ }^{21,22}$ and in mucosa of distal colon in rat after irradiation. ${ }^{13}$ GCs are secreted by mucosal cells and we have described corticosterone secretion by native MSCs. The relevance of intestinal GC synthesis in the regulation of immune cells has been demonstrated ${ }^{19}$ and experiments have established that inhibition of GC synthesis in mice aggravates colonic damage. ${ }^{36}$ We have also demonstrated the worsening of epithelial damage after inhibition of GC action after irradiation (Figure 7). It has been demonstrated that molecules involved in GC synthesis are also important regulators of intestinal crypt cell proliferation. ${ }^{37}$ The benefits of stem cell therapy rely on the combinatorial effect of several processes. The involvement of the GC pathway in the MSC immunosuppressive effect could therefore contribute to determining the potential link between the anti-inflammatory and regenerative abilities of MSCs. This hypothesis is in accordance with our results; indeed, blocking GC action with RU486 abolishes the benefits of MSCs on ulcerated mucosa. These results may be of importance for understanding the molecular mechanisms of the benefits of MSCs and could open up new perspectives for improving the therapeutic potential of MSC therapy, not only for radiation-induced inflammation but also for other inflammatory bowel diseases.

\section{METHODS}

Animals, treatments, sample collection, and immunohistochemistry. All experiments were performed in compliance with the French laws and guidelines for animal experimentation (P08-13). The $300 \mathrm{~g}$ wild-type male Sprague-Dawley rats were purchased from Charles River Laboratories (L'Arbresle, France). The animals were anesthetized by isoflurane inhalation and a single $27-G r a y$ dose was delivered by a ${ }^{60}$ Co source through a $2 \times 3 \mathrm{~cm}$ window centered on the colorectal region. ${ }^{12}$ Dose-scaling evaluation of the MSC effect was done. We injected $0.1,1,5$, or 10 millions of autologous MSCs in the tail vein of the rat (immediately after radiation exposure). We determined that an infusion of 5 million MSCs significantly improves the epithelial injury score and that an injection of 10 million MSCs does not increase the benefit any more. The animals were ethically killed at indicated times after radiation exposure. Cardiac puncture was made under anesthesia. The collected blood was transferred in EDTA sample collection tubes and centrifuged (2,500 r.p.m., $20 \mathrm{~min}$ ) to separate white blood cells from red cells and plasma. The MLNs and colorectum were sampled and transferred into calcium- and magnesium-free Hank's balanced salt solution (Invitrogen, Saint Aubin, France). For histological analysis, the colorectum was fixed in $4 \%$ formaldehyde embedded in paraffin and analyzed by immunohistochemistry as described in the Supplementary Materials online. For in vivo RU486 injection, the rats were injected subcutaneously every 3 days for 21 days at $40 \mathrm{mg} \mathrm{kg}^{-1}$ RU486 (Sigma, Saint Quentin Fallavier, France) and suspended in olive oil 1/10 ethanol (vehicle). Controls, irradiated, and irradiated-MSCtreated rats were injected with RU486 or vehicle alone.

MSC culture and stimulation. Bone marrow MSCs were obtained from the rat and the phenotype was verified as described in the Supplementary Materials. Their potential of differentiation was also evaluated as described by Rochefort et al. ${ }^{38}$ For in vitro MSC stimulation, analyses of the MSC genic expression were performed after $24 \mathrm{~h}$ of in vitro stimulation by $\mathrm{H}_{2} \mathrm{O}_{2}\left(10^{-8} \mathrm{M}\right)$ or synthetic TLR ligands from Invivogen: PamCSK4 (TLR2; $2.5 \mu \mathrm{g} \mathrm{ml}^{-1}$ ), UltraPure lipopolysaccharide (TLR4; $1 \mu \mathrm{g} \mathrm{ml}^{-1}$ ) and flagellin (TLR5; $2.5 \mu \mathrm{g} \mathrm{ml}^{-1}$ ) on $70 \%$ confluent cells. Cells were then trypsinized, washed in phosphatebuffered saline (Invitrogen) and frozen in liquid nitrogen.

Isolation of colonic leukocytes and flow cytometry analysis. Immediately after collection, the distal colon was cut into $5-\mathrm{mm}$ pieces and transferred into a solution of Hank's balanced salt solution containing 0.752 units $\mathrm{ml}^{-1}$ of Liberase TL (Roche Applied Science, Boulogne-Billancourt, France) and $0.1 \mathrm{mg} \mathrm{ml}^{-1}$ of DNase I (Roche Applied Science), and incubated at $37^{\circ} \mathrm{C}$ for four digestion rounds. The obtained cells were then settled on a Percoll gradient. The cells were suspended in a $40 \%$ Percoll solution and layered on a $100 \%$ Percoll phase before centrifugation (20 min, 2,300 r.p.m. without break). The leukocytes recovered were analyzed by flow cytometry (the antibody references are provided in the Supplementary Materials), and by using a FACS Canto II and DIVA software (BD Biosciences, Le Pont de Claix, France). The results are expressed as the percentage of positive cells among the total leukocyte population.

Sorting of CD4 and CD8 T lymphocytes. The separation of CD4 and CD8 T lymphocytes from draining MLNs was done using MACS technology (Miltenyi Biotech, Paris), according to the manufacturer's instructions, using LS columns. The cell purity was assessed by flow cytometry analysis and we obtained an average of $86.5 \pm 1.7 \%$ and $71.5 \pm 1.7 \%$ purity for CD4 and CD8 cells, respectively.

Lymphocyte proliferation. To measure lymphocytes proliferation, CD4 or CD8 lymphocytes were seeded in triplicate into 96-well cell culture plates (75,000 cells per well for CD4 and 150,000 cells per well for CD8) in RPMI 1640 medium (Invitrogen) supplemented with 10\% fetal bovine serum and $50 \mathrm{~mm} \beta$-mercaptoethanol (Sigma-Aldrich, Saint Quentin Fallavier, France). The lymphocytes were stimulated with $20 \mathrm{ng} \mathrm{ml}^{-1}$ of Phorbol 12-Myristate 13-Acetate (Sigma-Aldrich) and $5 \mu \mathrm{g} \mathrm{ml}^{-1}$ of Concanavalin A (Sigma-Aldrich). For mixed lymphocytes reaction, $7 \times 10^{5}$ stimulator lymphocytes $(15 \mathrm{~Gy}$ irradiated) were cultured with $7 \times 10^{5}$ responder lymphocytes with or without various doses of MSCs $\left(1 \times 10^{5}-1 \times 10^{4}\right.$ cells). RU486 (Sigma) was added to the reaction at $10^{-6} \mathrm{M}$ when indicated. After $72 \mathrm{~h}$, BrdU was added and we waited an additional $24 \mathrm{~h}$ to reveal cell proliferation by chromogenic assay (Roche Applied Science) according to the manufacturer's instructions.

Measurement of apoptosis on proliferating sorted lymphocytes. The EdU proliferation kit (Click-iT EdU Flow Cytometry Assay Kit; Invitrogen) was combined with flow cytometry apoptosis detection by Annexin V antibody (BD Biosciences). Cells $\left(5 \times 10^{5}\right)$ were seeded in a 96-well cell culture plate in lymphocyte culture medium with EdU. After $22 \mathrm{~h}$, cells were collected, washed, stained for anti-Annexin V expression and EdU incorporation, and analyzed by flow cytometry. The results are expressed as the percentage of Annexin V-positive cells among total EdU-proliferating cells.

Immunofluorescence. The CD4 and CD8 cells were purified from MLNs. Different conditions were analyzed: unstimulated cells, cells activated by anti-CD3 antibody during $15 \mathrm{~min}$ at $37^{\circ} \mathrm{C}$ (G4-18, BD Biosciences, $1 \mu \mathrm{g} \mathrm{ml}^{-1}$ ), cells treated by $1 \mu \mathrm{M}$ dexamethasone during $15 \mathrm{~min}$ at $37^{\circ} \mathrm{C}$ (Sigma-Aldrich), and cells treated by dexamethasone then activated by anti-CD3 antibody. The cells were fixed onto microscope slides and stained for TCR activation using antibody against the activated form of p56lck (phosphor-Tyr 394, Santa Cruz, Dallas, TX). MSCs were cultured on the Lab-Tek chamber slide system (Nunc, Sigma-Aldrich) and stained for 11 $\beta$-HSD1 enzyme (11 $\beta$-HSD1, Rabbit polyclonal; LSBio, Nantes, France) or control isotype (Dako, Trappes, France). Staining was revealed using a fluorescent secondary antibody (Goat anti-rabbit Alexa 568; 
Invitrogen) and DAPI (4',6-diamidino-2-phenylindole; Vectashield HardSet Mounting Medium with DAPI; Vector Laboratories, Burlingame, CA). Samples were scanned using a confocal microscope (LSM 780 NLO; Carl Zeiss MicroImaging, Jena, Germany) using a 405$\mathrm{nm}$ and a $637-\mathrm{nm}$ LASER under a $\times 20$ water objective.

Enzyme-linked immuno-assay and real-time quantitative PCR analyses. Colonic mucosa was separated from muscularis propria by gentle dissection. Mucosa protein extracts were obtained by tissue disruption in phosphate-buffered saline $1 \times$ with protease inhibitors (complete Mini; Roche, Boulogne-Billancourt, France) and total RNA was extracted from the mucosal samples with the RNeasy Mini kit (Qiagen, Courtaboeuf, France). The enzyme-linked immuno-assay and PCR analyses are described in detail in the Supplementary Materials.

Statistical analysis. The results are expressed as mean \pm s.e.m. Differences between groups were analyzed using the unpaired Student's $t$-test or non-parametric Mann-Whitney test when necessary. $P \leq 0.05$ was considered as statistically significant.

SUPPLEMENTARY MATERIAL is linked to the online version of the paper at http://www.nature.com/mi

\section{ACKNOWLEDGMENTS}

We thank Dr Agnes François, Dr Valérie Holler, and Jean-Victor LacavéLapalun for their scientific and technical support. We thank Patrick Laloi for critical reading of the manuscript. This work was supported by grants from the lle-de-France regional council.

\section{DISCLOSURE}

The authors declared no conflict of interest.

(c) 2014 Society for Mucosal Immunology

\section{REFERENCES}

1. Henson, C. Chronic radiation proctitis: issues surrounding delayed bowe dysfunction post-pelvic radiotherapy and an update on medical treatment. Therap Adv Gastroenterol 3, 359-365 (2010).

2. Andreyev, H.J., Wotherspoon, A., Denham, J.W. \& Hauer-Jensen, M Defining pelvic-radiation disease for the survivorship era. Lancet Oncol 11 310-312 (2010).

3. Theis, V.S., Sripadam, R., Ramani, V. \& Lal, S. Chronic radiation enteritis. Clin Oncol (R Coll Radiol) 22, 70-83 (2010).

4. Wedlake, L.J., Thomas, K., Lalji, A., Blake, P. \& Khoo, V.S. et al. Predicting late effects of pelvic radiotherapy: is there a better approach? Int $J$ Radiat Oncol Biol Phys 78, 1163-1170 (2010).

5. Gonzalez, M.A., Gonzalez-Rey, E., Rico, L., Buscher, D. \& Delgado, M. Adipose-derived mesenchymal stem cells alleviate experimental colitis by inhibiting inflammatory and autoimmune responses. Gastroenterology 136, 978-989 (2009).

6. Lim, J.H., Kim, J.S., Yoon, I.H., Shin, J.S. \& Nam, H.Y. et al. Immunomodulation of delayed-type hypersensitivity responses by mesenchymal stem cells is associated with bystander T cell apoptosis in the draining lymph node. J Immunol 185, 4022-4029 (2010).

7. Ren, G., Zhang, L., Zhao, X., Xu, G. \& Zhang, Y. et al. Mesenchymal stem cell-mediated immunosuppression occurs via concerted action of chemokines and nitric oxide. Cell Stem Cell 2, 141-150 (2008).

8. Zappia, E., Casazza, S., Pedemonte, E., Benvenuto, F. \& Bonanni, I. et al. Mesenchymal stem cells ameliorate experimental autoimmune encephalomyelitis inducing T-cell anergy. Blood 106, 1755-1761 (2005).

9. Le Blanc, K., Rasmusson, I., Sundberg, B., Götherström, C. \& Hassan, M. et al. Treatment of severe acute graft-versus-host disease with third party haploidentical mesenchymal stem cells. Lancet 363, 1439-1441 (2004).

10. Karussis, D., Karageorgiou, C., Vaknin-Dembinsky, A., Gowda-Kurkalli, B. \& Gomori, J.M. et al. Safety and immunological effects of mesenchymal stem cell transplantation in patients with multiple sclerosis and amyotrophic lateral sclerosis. Arch Neurol 67, 1187-1194 (2010).
11. Sun, L., Wang, D., Liang, J., Zhang, H. \& Feng, X. et al. Umbilical cord mesenchymal stem cell transplantation in severe and refractory systemic lupus erythematosus. Arthritis Rheum 62, 2467-2475 (2010).

12. Jullien, N., Blirando, K., Milliat, F., Sabourin, J.C. \& Benderitter, M. et al. Up-regulation of endothelin type a receptor in human and rat radiation proctitis: preclinical therapeutic approach with endothelin receptor blockade. Int J Radiat Oncol Biol Phys 74, 528-538 (2009).

13. Semont, A., Demarquay, C., Bessout, R., Durand, C. \& Benderitter, M. et al. Mesenchymal stem cell therapy stimulates endogenous host progenitor cells to improve colonic epithelial regeneration. PLoS One 8, e70170 (2013).

14. Blirando, K., Milliat, F., Martelly, I., Sabourin, J.C. \& Benderitter, M. et al. Mast cells are an essential component of human radiation proctitis and contribute to experimental colorectal damage in mice. Am J Pathol 178, 640-651 (2011).

15. Linard, C., Marquette, C., Clarençon, D., Galonnier, M., Mathieu, J. \& Pennequin, A. et al. Acute ileal inflammatory cytokine response induced by irradiation is modulated by subdiaphragmatic vagotomy. Journal of Neuroimmunology 168, 83-95 (2005).

16. Gonzalez, M.A., Gonzalez-Rey, E., Rico, L., Buscher, D. \& Delgado, M. Treatment of experimental arthritis by inducing immune tolerance with human adipose-derived mesenchymal stem cells. Arthritis Rheum 60, 1006-1019 (2009).

17. Parekkadan, B., Upadhyay, R., Dunham, J., Iwamoto, Y. \& Mizoguchi, E. et al. Bone marrow stromal cell transplants prevent experimental enterocolitis and require host CD11b + splenocytes. Gastroenterology 140, 966-975 (2011).

18. Parekkadan, B., Tilles, A.W. \& Yarmush, M.L. Bone marrow-derived mesenchymal stem cells ameliorate autoimmune enteropathy independently of regulatory T cells. Stem Cells 26, 1913-1919 (2008).

19. Cima, I., Corazza, N., Dick, B., Fuhrer, A. \& Herren, S. et al. Intestinal epithelial cells synthesize glucocorticoids and regulate $\mathrm{T}$ cell activation. J Exp Med 200, 1635-1646 (2004).

20. Lowenberg, M., Verhaar, A.P., Bilderbeek, J., Marle, J. \& Buttgereit, F. et al. Glucocorticoids cause rapid dissociation of a T-cell-receptor-associated protein complex containing LCK and FYN. EMBO Rep 7, 1023-1029 (2006).

21. Francois, S., Bensidhoum, M., Mouiseddine, M., Mazurier, C. \& Allenet, B. et al. Local irradiation not only induces homing of human mesenchymal stem cells at exposed sites but promotes their widespread engraftment to multiple organs: a study of their quantitative distribution after irradiation damage. Stem Cells 24, 1020-1029 (2006).

22. Semont, A., Mouiseddine, M., Francois, A., Demarquay, C. \& Mathieu, N. et al. Mesenchymal stem cells improve small intestinal integrity through regulation of endogenous epithelial cell homeostasis. Cell Death Differ 17, 952-961 (2010).

23. Meirelles Lda, S., Fontes, A.M., Covas, D.T. \& Caplan, A.I. Mechanisms involved in the therapeutic properties of mesenchymal stem cells. Cytokine Growth Factor Rev 20, 419-427 (2009).

24. Krampera, M., Cosmi, L., Angeli, R., Pasini, A. \& Liotta, F. et al. Role for interferon-gamma in the immunomodulatory activity of human bone marrow mesenchymal stem cells. Stem Cells 24, 386-398 (2006).

25. Gremy, O., Benderitter, M. \& Linard, C. Acute and persisting Th2-like immune response after fractionated colorectal gamma-irradiation. World $\mathrm{J}$ Gastroenterology 14, 7075-7085 (2008).

26. Garg, S., Boerma, M., Wang, J., Fu, Q. \& Loose, D.S. et al. Influence of sublethal total-body irradiation on immune cell populations in the intestinal mucosa. Radiat Res 173, 469-478 (2010).

27. Billiard, F., Buard, V., Benderitter, M. \& Linard, C. Abdominal gammaradiation induces an accumulation of function-impaired regulatory Tcells in the small intestine. Int J Radiat Oncol Biol Phys 80, 869-876 (2011).

28. Reits, E.A., Hodge, J.W., Herberts, C.A., Groothuis, T.A. \& Chakraborty, M. et al. Radiation modulates the peptide repertoire, enhances MHC class I expression, and induces successful antitumor immunotherapy. J Exp Med 203, 1259-1271 (2006).

29. Takeshima, T., Chamoto, K., Wakita, D., Ohkuri, T. \& Togashi, Y. et al. Local radiation therapy inhibits tumor growth through the generation of tumorspecific CTL: its potentiation by combination with Th1 cell therapy. Cancer Res 70, 2697-2706 (2010). 
30. Friedman, E.J. Immune modulation by ionizing radiation and its implications for cancer immunotherapy. Curr Pharm Des 8, 1765-1780 (2002).

31. Ringdén, O., Uzunel, M., Rasmusson, I., Remberger, M. \& Sundberg, B. et al. Mesenchymal stem cells for treatment of therapy-resistant graftversus-host disease. Transplantation 81, 1390-1397 (2006).

32. Garcia-Olmo, D., Garcia-Arranz, M., Herreros, D., Pascual, I. \& Peiro, C. et al. A phase I clinical trial of the treatment of Crohn's fistula by adipose mesenchymal stem cell transplantation. Dis Colon Rectum 48, 1416-1423 (2005).

33. Uccelli, A., Moretta, L. \& Pistoia, V. Mesenchymal stem cells in health and disease. Nat Rev Immunol 8, 726-736 (2008).

34. Nemeth, K., Leelahavanichkul, A., Yuen, P.S., Mayer, B. \& Parmelee, A. et al. Bone marrow stromal cells attenuate sepsis via prostaglandin
$E(2)$-dependent reprogramming of host macrophages to increase their interleukin-10 production. Nat Med 15, 42-49 (2009).

35. Yagi, H., Soto-Gutierrez, A., Parekkadan, B., Kitagawa, Y. \& Tompkins, R.G. et al. Mesenchymal stem cells: Mechanisms of immunomodulation and homing. Cell Transplant 19, 667-679 (2010).

36. Coste, A., Dubuquoy, L., Barnouin, R., Annicotte, J.S. \& Magnier, B. et al. LRH1-mediated glucocorticoid synthesis in enterocytes protects against inflammatory bowel disease. Proc Natl Acad Sci U S A. 104, 13098-13103 (2007).

37. Botrugno, O.A., Fayard, E., Annicotte, J.S., Haby, C. \& Brennan, T. et al. Synergy between $\mathrm{LRH}-1$ and beta-catenin induces $\mathrm{G} 1$ cyclin-mediated cell proliferation. Mol Cell 15, 499-509 (2004).

38. Rochefort, G.Y., Delorme, B., Lopez, A., Herault, O. \& Bonnet, P. et al. Multipotential mesenchymal stem cells are mobilized into peripheral blood by hypoxia. Stem Cells 24, 2202-2208 (2006). 